\title{
Composite Hermite Curves for Time-Based Aircraft Spacing at Meter Fix
}

\author{
Thierry Miquel* \\ Direction des Services de la Navigation Aérienne, Toulouse, FRANCE \\ Félix Mora-Camino ${ }^{\dagger}$ \\ Ecole Nationale de l'Aviation Civile and LAAS du CNRS, Toulouse, France \\ and \\ Jean-Marc Loscos ${ }^{\star}$ \\ Direction des Services de la Navigation Aérienne, Toulouse, FRANCE
}

\begin{abstract}
The delegation to the flight crew of some tasks currently performed by air traffic controllers provides new perspectives potentially to increase air traffic control efficiency. More specifically, the task of establishing properly spaced landing sequences is very demanding in heavy traffic conditions for the air traffic controllers in charge of the terminal maneuvering area. Automatic merging operations could relieve air traffic controllers of providing time-consuming radar-vectoring instructions. The outcomes of this paper are twofold: firstly composite Hermite curves to generate a path with length and endpoint constraints are presented. This reference trajectory is used to stretch the path of an airliner in order to delay its arrival on a meter fix chosen by air traffic control. Secondly, the design of a nonlinear control law based on feedback linearization to track the reference trajectory is presented. This communication treats level flight, but wind is considered. The design is followed by two illustrative examples which show the effectiveness of the proposed approach.
\end{abstract}

\section{Introduction}

$\mathrm{T}$ he overall goal of the R\&D community has always been to improve Air Traffic Management (ATM) operations through a greater involvement of pilots in cooperation with air traffic controllers. The technology called ADS-B (Automatic Dependent Surveillance-Broadcast) is now available for civil aircraft ${ }^{1}$ to enhance flight crew air traffic situational awareness. In addition, the Airborne Separation Assistance System (ASAS) concept was proposed to ICAO in March $1995^{2}$ to take advantage of the airborne capabilities in particular for communication, navigation and surveillance. The range of ASAS applications is very wide ${ }^{3}$ : those applications are designed to be fully integrated into ATM operations, and most of them involve the active cooperation of air traffic controllers and pilots.

By ICAO definition ${ }^{4}$ ASAS is an airborne system that allows the flight crew to maintain separation between their aircraft and one or more other aircraft, and provides information concerning the surrounding traffic. ASAS on board an aircraft interacts with the existing systems (particularly the Flight Management System - FMS) or even with the installed equipment (particularly the display screens); this may also require new equipment depending on the architecture needed. Not only the system, but also its utilization was defined by ICAO: an ASAS application is a set of operational procedures for air traffic controllers and flight crews, which makes use of the capabilities of airborne separation assistance systems to meet a clearly-defined operational objective.

This paper considers 'Airborne Spacing' applications. In this category of ASAS applications for maintaining spacing (without transfer of responsibility) the flight crews must acquire and maintain a given distance or time with another designated aircraft, after a new Air Traffic Control (ATC) instruction. Even if the flight crews inherit new

\footnotetext{
* PhD, Civil Aviation Design and Operation Engineer, Separation and Alerting Systems Unit, miquel@cena.fr

† Senior Researcher at LAAS du CNRS, Professor of Automatic Control and Avionics at Ecole Nationale de 1'Aviation civile, Felix.MORA@enac.fr

* Civil Aviation Design and Operation Engineer, Head of Separation and Alerting Systems Unit, loscos@cena.fr Copyright $@ 2007$ by DSNA. Published by the American Institute of Aeronautics and Astronautics, Inc., with permission.
} 
tasks, the separation between aircraft is still the controller's responsibility. This ASAS application can thus be regarded as a new instruction based on the aircraft's new capabilities, on the controller's initiative. Amongst the various applications considered, 'Sequencing \& Merging' appears to be promising for sequencing aircraft that arrive at large terminal maneuvering areas (TMA).

The task of establishing properly spaced landing sequences is very demanding in heavy traffic conditions for the air traffic controllers in charge of the terminal maneuvering area. With the sustained increase of air traffic leading to airspace saturation, new flight maneuvering capabilities are expected for commercial aircraft. Among these, automatic path stretching and tracking onboard airliners to achieve time-based separation at given meter fix appears as a promising solution to ease air traffic controllers' workload. Indeed, the envisioned path stretching maneuver could relieve air traffic controllers of the need to provide time-consuming radar-vectoring instructions. It is realized onboard the aircraft using their sensing and communication facilities, in a decentralized way.

This paper focuses on path stretching and tracking for time-Based aircraft spacing at meter fix. The path stretching problem consists in finding curves that satisfy constraints on the positions and tangents at the two endpoints. Finding a smooth path with continuous curvature that can match arbitrary endpoint constraints and length constraint is nontrivial. Several techniques can be used, including geometric approaches ${ }^{5,6,7}$, the use of optimal control $^{8,9}$ as well as evolutionary algorithms ${ }^{10}$. In that paper, a geometric approach using composite Hermite curves is followed.

The paper is organized as follows: in the next section, the path stretching planner based on composite Hermite curves to generate a path with length and endpoint constraints is described. This reference trajectory is used to stretch the path of an airliner in order to delay its arrival on a meter fix chosen by air traffic control. Then, the design of a nonlinear control law based on feedback linearization to track the reference trajectory is presented. The design is followed by two illustrative examples which treats level flight but where wind is considered. Finally, conclusions on the effectiveness of the proposed approach are drawn.

\section{PATH STRETCHING}

\section{A. Preliminaries}

In the following, a fully coordinated aircraft (i.e. side-slip angle is assumed to be zero) flying over a flat and nonrotating earth within standard atmosphere is assumed. Furthermore, and as in many modern jets, it is assumed in the following that a Flight Management System (FMS) and an autopilot function dealing with heading control is available onboard the trailing aircraft.

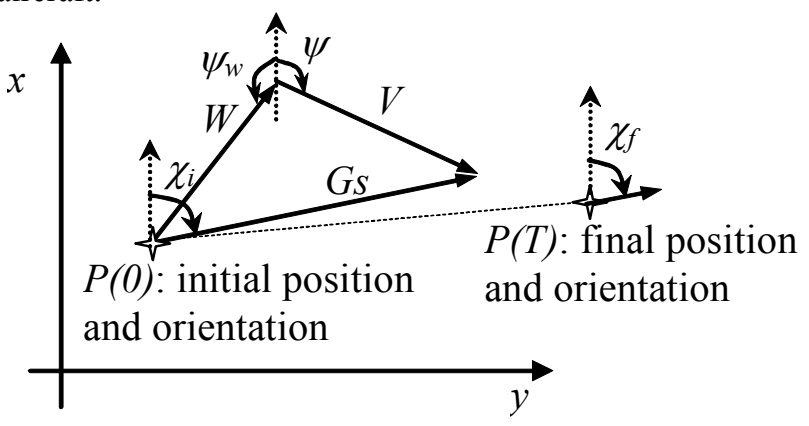

Fig. 1: Speed triangle

As a consequence, the kinematics equations of motion of the aircraft are the following (see Fig. 1):

$$
\left\{\begin{array}{l}
\dot{x}=V \cos (\psi)-W \cos \left(\psi_{w}\right)=G s \cos (\chi) \\
\dot{y}=V \sin (\psi)-W \sin \left(\psi_{w}\right)=G s \sin (\chi)
\end{array}\right.
$$

In the preceding equations $\psi_{w}$ is the direction from where the wind is blowing, $W$ is the speed of the wind, $\psi$ the heading of the aircraft and $V$ its airspeed, $G s$ the groundspeed and $\chi$ the track angle.

In order to be independent from wind, the path stretching planner generates an airborne open loop trajectory for the following system, where $\left(x_{a}(0), y_{a}(0), \psi_{a}(0)\right)$ and $\left(x_{a}(T), y_{a}(T), \psi_{a}(T)\right)$ are given constraints at the beginning and the end of the trajectory:

$$
\left\{\begin{array}{l}
\dot{x}_{a}=V \cos \left(\psi_{a}\right) \\
\dot{y}_{a}=V \sin \left(\psi_{a}\right)
\end{array}\right.
$$




\section{B. Composite Hermite curves}

The objective for the path planning is to stretch the trajectory while maintaining a smooth motion. The problem consists of generating a smooth trajectory curve by joining two distinct configurations, the oriented start and end points, with a constraint on the time at which end point is reached.

In this paper composite Hermite curve are used ${ }^{11}$. Two piecewise $C^{2}$-continuous segments are used. Each segment is a cubic polynomial Hermite curve $\mathbf{Q}(s), s \in\left[s_{0}, s_{l}\right]$ where $s_{0}, s_{1} \in \mathfrak{R}$ and $s_{0}<s_{1}$ satisfy the following endpoint location and tangent vector conditions:

$$
\begin{array}{ll}
\mathbf{Q}\left(s_{0}\right)=\mathbf{P}_{0}, & \mathbf{Q}^{\prime}\left(s_{0}\right)=a_{0} \mathbf{V}_{0} \\
\mathbf{Q}\left(s_{1}\right)=\mathbf{P}_{1}, & \mathbf{Q}^{\prime}\left(s_{1}\right)=a_{1} \mathbf{V}_{1}
\end{array}
$$

Points $\mathbf{P}_{0}$ and $\mathbf{P}_{1}$ are given $2 \mathrm{D}$ (or $3 \mathrm{D}$ ) points, and $\mathbf{V}_{0}$ and $\mathbf{V}_{1}$ are given tangent vectors at $\mathbf{P}_{0}$ and $\mathbf{P}_{1}$ respectively. $\mathbf{Q}(s)$ can be expressed as follows:

Where

$$
\begin{aligned}
& \mathbf{Q}(s)=(2 \tau+1)(\tau-1)^{2} \mathbf{P}_{0}+(-2 \tau+3) \tau^{2} \mathbf{P}_{1} \\
& +(1-\tau)^{2} \tau\left(s_{1}-s_{0}\right) a_{0} \mathbf{V}_{0}+(\tau-1) \tau^{2}\left(s_{1}-s_{0}\right) a_{1} \mathbf{V}_{1}
\end{aligned}
$$

$$
s=V \cdot t, s_{0}=0, s_{1}=V \cdot T, \tau=\frac{s-s_{0}}{s_{1}-s_{0}}=\frac{t}{T}
$$

A negative value of $a_{0}$ or $a_{1}$ means that the tangent vector of the Hermite curve is in the opposite direction of $\mathbf{V}_{0}$ or $\mathbf{V}_{1}$.

\section{Open loop trajectory}

The open loop trajectory is obtained through a composite Hermite curve composed by segment $P(0) P i$ and segment $P i P(T)$ as depicted in Figure 2. $\left(P(0), \chi_{0}\right)$ represents the starting position and orientation of the aircraft whereas $\left(P(T), \chi_{T}\right)$ represents the final position and orientation of the aircraft:

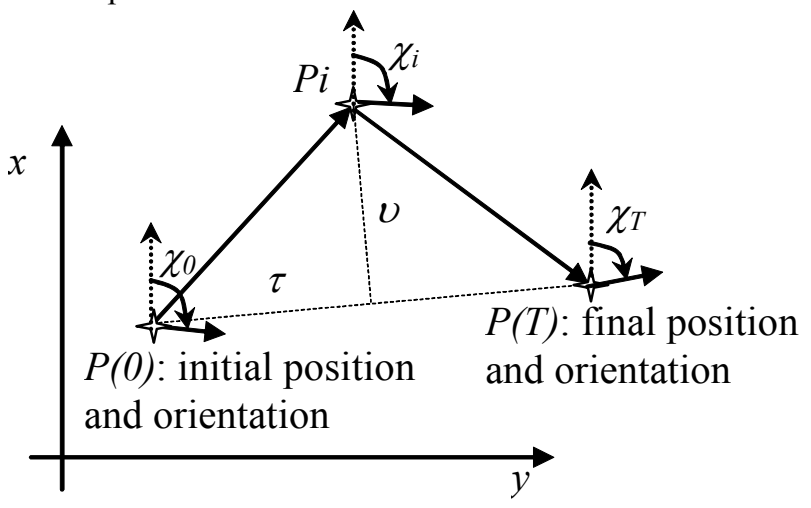

Fig. 2: Parameterization of composite Hermite curves

Intermediate point $P i$ is positioned to the line perpendicular to the segment $P(0) P(T)$, where $P(0)$ designates the initial position of the aircraft, and $P(T)$ its desired position after $T$ time of flight. Required time $T$ is supposed to be given by air traffic control through the help of an Arrival Manager (AMAN)

Denoting by $\vec{v}$ the vector perpendicular to segment $P(0) P(T)$, vector $\overrightarrow{O P i}$ is defined by:

$$
\overrightarrow{O P i}=\vec{\tau}+\vec{v} \text { where } \vec{\tau}=\frac{P(0) P(T)}{2}
$$

Orientation $\chi_{i}$ of intermediate point $P i$ is taken to be the angle of the vector bisecting segment $P(0) P i$ and segment $P i P(T)$.

Let $L$ be the desired length of the Composite Hermite curve. The desired length $L=V \cdot T$ is achieved by setting appropriately the length of vector $\vec{v}$ perpendicular to segment $P(0) P(T)$.

\section{Path Tracking}

The purpose of path tracking is to make the aircraft able to follow the reference trajectory established by the path stretching planner. 
The control of the lateral motion of the trailing aircraft to follow the reference trajectory is achieved by providing heading cues to the autopilot. It is based on feedback linearization ${ }^{12}$ which allows the transformation of a nonlinear system into an equivalent linear system.

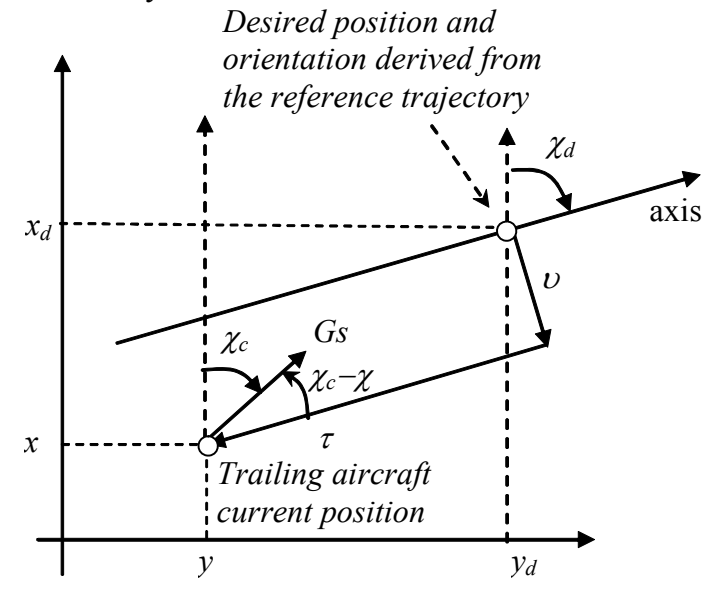

Fig. 3: Cross track distance $\boldsymbol{v}$ and the along track distance $\tau$ between current and desired position

From Figure 3, the cross track distance $v$ and the along track distance $\tau$ from the current position $(x, y)$ of the aircraft to the axis oriented by the desired track $\chi_{d}$ and passing through the desired position $\left(x_{d}, y_{d}\right)$ are given by:

$$
\left[\begin{array}{l}
\tau \\
v
\end{array}\right]=\left[\begin{array}{cc}
\cos \left(\chi_{d}\right) & \sin \left(\chi_{d}\right) \\
-\sin \left(\chi_{d}\right) & \cos \left(\chi_{d}\right)
\end{array}\right] \cdot\left[\begin{array}{c}
x-x_{d} \\
y-y_{d}
\end{array}\right]
$$

In addition, the dynamics of the cross track distance $v$ is given by:

$$
\frac{d}{d t} v=G s \cdot \sin \left(\chi_{c}-\chi_{d}\right)
$$

Now, let us impose a first order dynamic for the cross track distance:

$$
\frac{d}{d t} v=-\lambda \cdot v
$$

Combining the two previous equations leads to the expression for the commanded track angle $\chi_{c}$ :

$$
-\lambda \cdot v=G s \cdot \sin \left(\chi_{c}-\chi_{d}\right) \Leftrightarrow \chi_{c}=\chi_{d}-\arcsin \left(\frac{\lambda \cdot v}{G s}\right)
$$

Previous equation shows that the ratio $\lambda \cdot v / G s$ shall lie between -1 and +1 . In addition, the difference between the desired track $\chi_{d}$ and the commanded track $\chi_{c}$ shall be limited between $-\pi / 2$ and $+\pi / 2$.

The previous equation associated with (1) leads to the expression of the commanded heading angle $\psi_{c}$ :

$$
\left\{\begin{array}{l}
\psi_{c}=\arctan \left(\frac{G s \cdot \sin \left(\chi_{c}\right)+W \cdot \sin \left(\psi_{w}\right)}{G s \cdot \cos \left(\chi_{c}\right)+W \cdot \cos \left(\psi_{w}\right)}\right)=\arctan \left(\frac{G s \cdot \sin \left(\chi_{d}-\arcsin \left(\frac{\lambda \cdot v}{G s}\right)\right)+W \cdot \sin \left(\psi_{w}\right)}{G s \cdot \cos \left(\chi_{d}-\arcsin \left(\frac{\lambda \cdot v}{G s}\right)\right)+W \cdot \cos \left(\psi_{w}\right)}\right) \\
G s=\sqrt{V^{2}+W^{2}-2 \cdot V \cdot W \cdot \cos \left(\psi_{d}-\psi_{w}\right)}
\end{array}\right.
$$

In the following, the ratio between the airspeed $V$ and parameter $\lambda$ has been set to the radius of turn $R$ of the aircraft:

$$
\frac{V}{\lambda}=R=\frac{V^{2}}{g \cdot \tan \left(\varphi_{\max }\right)} \Rightarrow \lambda=\frac{g \cdot \tan \left(\varphi_{\max }\right)}{V}
$$

Assuming a maximum bank angle $\varphi_{\max }$ of 30 degrees and an airspeed $V$ of $149 \mathrm{~m} / \mathrm{s}$, this leads to a value of parameter $\lambda$ at $0.038 \mathrm{sec}^{-1}$. 


\section{Illustrative examples}

\section{A. Scenarios}

In this section, two situations are presented in order to evaluate the properties of the path stretching and tracking control previously described.

The aircraft models which have been used is a fictitious commercial aircraft model (called RCAM) which was developed by GARTEUR - Group for Aeronautical Research and Technology in Europe (Magni, et al., 1997). The aircraft mass has been set to $120000 \mathrm{~kg}$. The airspeed (TAS) of the aircraft has been chosen to be $289 \mathrm{knots}$ (149 $\mathrm{m} / \mathrm{s}$ ) which corresponds to a conventional airspeed (CAS) of 250 knots at flight level 100. This airspeed is compliant with current procedure and observations on radar data.

The scenario is depicted in Fig. 4, and is built around real life meter fixes of an air navigation procedure in France. The leading aircraft starts its trajectory 40 NM before meter fix SOKMU, whereas the RCAM aircraft starts its trajectory vertical to meter fix DPE (Dieppe). It firstly converges towards meter fix SOKMU and then moves towards meter fix MERUE.

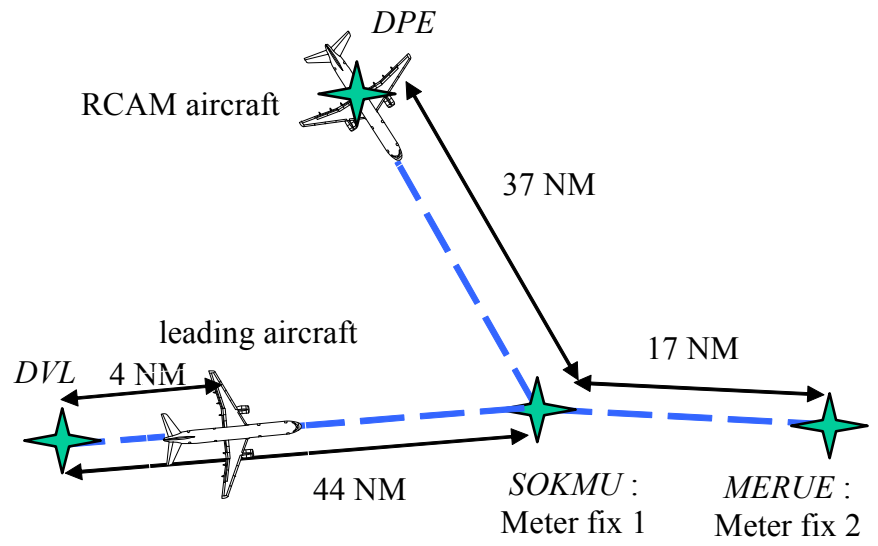

Fig. 4: Case study scenario

In the first situation, no wind is assumed. When no path stretching is applied, the leading aircraft passes meter fix SOKMU at $\mathrm{t}=497 \mathrm{sec}$ whereas the RCAM aircraft passes meter fix SOKMU before the leading aircraft at $\mathrm{t}=456 \mathrm{sec}$.

In the second situation, a wind of 97 knots $(50 \mathrm{~m} / \mathrm{s})$ blowing from East is assumed. When no path stretching is applied, the leading aircraft passes meter fix SOKMU at $\mathrm{t}=748 \mathrm{sec}$ whereas the RCAM aircraft passes meter fix SOKMU before the leading aircraft at $\mathrm{t}=538 \mathrm{sec}$.

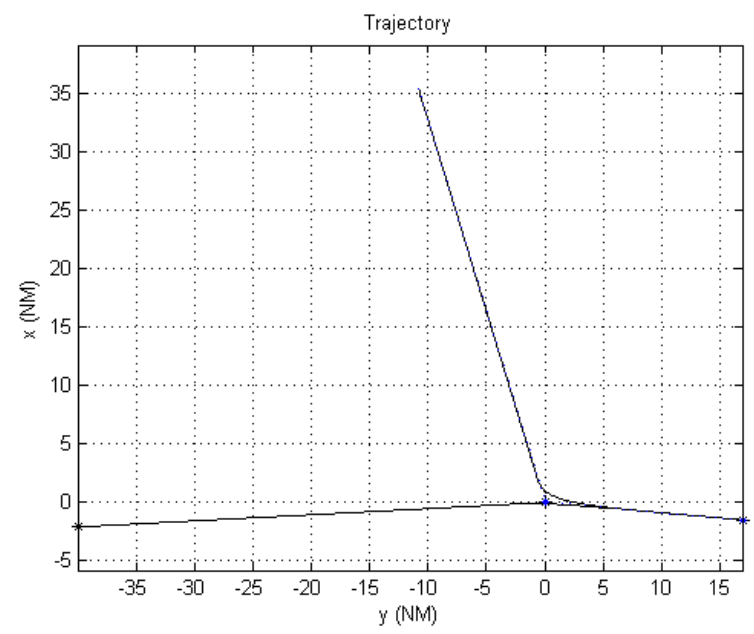

Fig. 5: Trajectory without path stretching

The objective of the simulations is to delay the arrival time of the RCAM aircraft to SOKMU by $90 \mathrm{sec}$. In the following, parameters $a_{0}$ and $a_{1}$ in (4) have been set to 50 . 


\section{B. Results when no wind is considered}

The estimated time at which the leading aircraft is at closest distance of SOKMU is obtained by dividing the distance between the aircraft and SOKMU by its ground speed, which gives $497 \mathrm{sec}$ (that is 40 NM divided by 149 $\mathrm{m} / \mathrm{s})$. Then the desired delay of $90 \mathrm{sec}$ is added. As a consequence, the estimated time $T$ used in (5) is $497+90=$ $587 \mathrm{sec}$. This leads to the desired length $L=V \cdot T$ of the Composite Hermite curve, where $V$ is the airspeed of the RCAM aircraft, which is $149 \mathrm{~m} / \mathrm{s}$. To achieve that length, the norm of vector $\vec{v}$ in (6) shall be set to $12.76 \mathrm{NM}$.

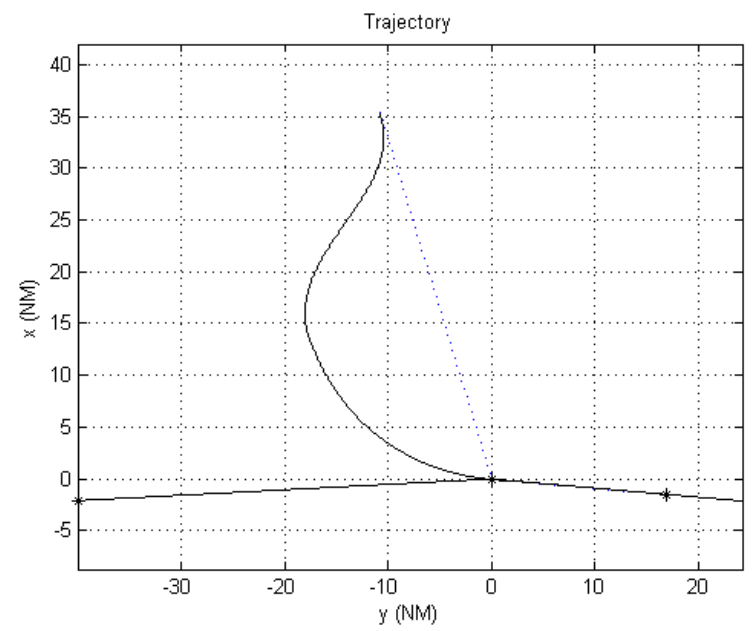

Fig. 6: Trajectory with path stretching and no wind

The simulation shows that the actual time at which the leading aircraft is at closest distance of SOKMU is 497 $\mathrm{sec}$, whereas the RCAM aircraft is at closest distance of SOKMU at $589 \mathrm{sec}$ : the time difference is $92 \mathrm{sec}$, that is +2 sec of error compared with the desired delay of $90 \mathrm{sec}$.

\section{Results when wind is considered}

In that situation, a wind of 97 knots $(50 \mathrm{~m} / \mathrm{s})$ blowing from East is assumed, but the same methodology than is the previous section is applied.

The estimated time at which the leading aircraft is at closest distance of SOKMU is obtained by dividing the distance between the aircraft and SOKMU by its ground speed, which gives $748 \mathrm{sec}$ (that is 40 NM divided by 99 $\mathrm{m} / \mathrm{s})$. Then the desired delay of $90 \mathrm{sec}$ is added. As a consequence, the estimated time $T$ used in (5) is $748+90=$ $838 \mathrm{sec}$. This leads to the desired length $L=V \cdot T$ of the Composite Hermite curve, where $V$ is the airspeed of the RCAM aircraft, which is $149 \mathrm{~m} / \mathrm{s}$. To achieve that length, the norm of vector $\vec{v}$ in (6) shall be set to $22.16 \mathrm{NM}$.

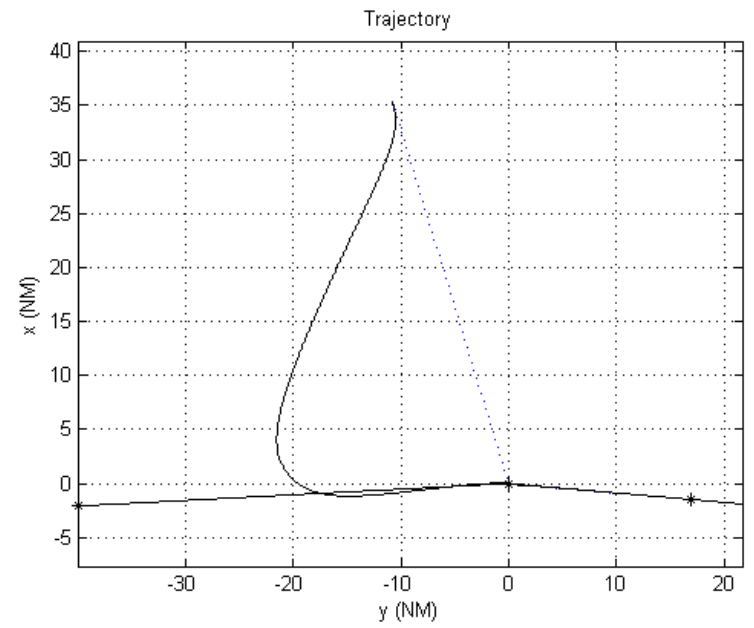

Fig. 6: Trajectory with path stretching and wind of $50 \mathrm{~m} / \mathrm{s}$ 
The simulation shows that the actual time at which the leading aircraft is at closest distance of SOKMU is 748 sec, whereas the RCAM aircraft is at closest distance of SOKMU at $837 \mathrm{sec}$ : the time difference is 89 sec, that is -1 $\mathrm{sec}$ of error compared with the desired delay of $90 \mathrm{sec}$.

\section{Conclusion}

In this paper, the design of a new FMS function dedicated to path stretching has been considered. This new function aims at achieving a specified delay between commercial aircraft at a specified meter fix.

This envisioned new capability onboard commercial aircraft takes advantage of the Automatic Dependent Surveillance-Broadcast $(A D S-B)$ and provides new perspectives to potentially increase air traffic control efficiency. It could be seen as the airborne counterpart of the ground based arrival manager (AMAN).

In this paper, the path stretching is based on composite Hermite curves to generate a path with length and endpoint constraints, whereas the tracking of the reference trajectory is made through heading control which is based on feedback linearization. This allows the trailing aircraft to accurately follow the reference trajectory. Airspeed is maintained at a constant value in order to avoid throttle demand.

Simulation results illustrate the efficiency of the proposed design. Nevertheless, additional studies in terms of operational scenarios, which take into account the vertical motion of the aircraft, are needed in order to refine and validate the proposed design. Future work will focus on the assessment of the proposed design using a set of numerous simulated encounters for the purpose of statistical evaluation. In addition adaptive path stretching will be addressed in order to take into account possible airspeed variations.

\section{Acknowledgments}

The authors wish to thank Philippe Louyot for helpful inputs and comments.

\section{References}

1 The Eleventh ICAO Air Navigation Conference, Automatic dependent surveillance - broadcast (Concept of Use), WP6 Item 1.2 in the list of documentation AN-Conf11 (2003)

(http://www.icao.int/icao/en/anb/meetings/anconf11/index.html)

2 Air Traffic Control Quarterly, Volume 13 Number 2 Special Issue on ASAS, Francis Casaux Editor (2005)

3 Loscos J.M., ASAS: towards new cooperation based on airborne separation, Revue technique de la DTI no. 2 , pp. 69-82 (2005)

4 The Eleventh ICAO Air Navigation Conference, Circular on airborne separation assistance system (ASAS), IP5 Item 1.2 in the list of documentation AN-Conf11 (2003) (http://www.icao.int/icao/en/anb/meetings/anconfl1/index.html)

5 Yamamo M., Iwamura M., Mohri A., Quasic-Time-Optimal Motion Planning of Mobile Platforms in the Presence of Obstacles, International Conference on Robotics and Automation, pp. 739-744 (1999)

6 Fraichard Th., Ahuactzin J. M., Smooth Path Planning for Cars, IEEE International Conference On Robotics and Automation May, pp. 21-26 (2001)

7 Lamiraux F., Kavraki L. E., Planning paths for elastic objects under manipulation constraints, International Journal of Robotics Research, vol. 20, no. 3, pp. 188-208 (2001)

8 Hagelauer, Mora-Camino, Evaluation of Practical Solutions for On Board Aircraft Fourth Dimensional Guidance, Journal of Guidance, Control and Dynamics, Vol 20(5), pp.1052-1054, 1997

9 Bertsekas, Dynamic Programming and Optimal Control, Vol .1, Athena Scientific, Belmont, Massachusetts, 1995

10 Gianazza D., Durand N., Assessment of the 3D-separation of Air Traffic Flows, 6th USA/Europe Seminar on Air Traffic Management Research and Development, Baltimore, June 2005

11 Yong, J.H., Cheng F. (2004). Geometric Hermite curves with minimum strain energy, Computer Aided Geometric Design, 21, 281-301

12 Khalil H.K., Nonlinear Systems, Third Edition, Prentice Hall, 2002

13 Magni J.F., Bennani S., Terlouw J., Robust Flight Control : A Design Challenge, Springer-Verlag Publications (1997) 